\title{
Melanin-Based Iridescent Feather Color in the Jungle Crow
}

\author{
Eunok LEE ${ }^{1,2)}$, Hideyuki TANAKA ${ }^{3)}$, Kazumasa WAKAMATSU ${ }^{4)}$ and Shoei SUGITA ${ }^{1)}$ \\ ${ }^{1)}$ Laboratory of Function and Morphology, Department of Animal Science, Faculty of Agriculture, Utsunomiya University, Utsunomiya \\ 321-8505, 2) United Graduate School of Agricultural Science, Tokyo University of Agriculture and Technology, Tokyo 183-8509, \\ ${ }^{3}$ Applied Biochemical Science, Faculty of Agriculture, Utsunomiya University, Utsunomiya 321-8505 and \\ ${ }^{4)}$ Department of Chemistry, Fujita Health University School of Health Sciences, Toyoake, Aichi 470-1192, Japan
}

(Received 20 January 2009/Accepted 29 May 2009)

ABSTRACT. Sexual differences in Japanese Jungle Crows (Corvus macrorhynchos) are not obvious because both sexes have black plumage. However, closer examination reveals that their plumage color is not only black; it is also iridescent color. Furthermore, the iridescence is more pronounced in adult males than that in females. The iridescence seems to be related to the density and concentration of melanin granules. The density of melanin granule and the concentration of eumelanin in feathers were significantly higher in males than that in females $(P<0.01)$. However, the amount of pheomelanin was below the detection limits in this study.

KEY WORDS: crow, feather color, HPLC, melanin.

Melanin is the most abundant and widespread pigment in birds. The 2 basic types of melanin pigments are eumelanin and pheomelanin [8]. Black and gray feathers are typically thought to contain mostly eumelanins, whereas earth-toned feathers are dominated by pheomelanins, much like red human hair [1]. The relationship between feather color and melanin pigments has been studied by various researchers. Hasse reported [2] melanin concentrations in wild rock pigeons (Columba livia) and domestic pigeons. McGraw and Wakamatsu measured melanin concentrations of zebra finches (Taeniopygia guttata) feathers color [6], and also reported melanin concentrations in the feathers both sexes of European barn swallows (Hirundo rustica rustica) [7].

Although, jungle crows (Corvus macrorhyncho) have melanin-based iridescent black feathers coloration, it's not known as to how much they have the melanin concentration and whether they have sexual differences in the amount of melanin concentration and density. The present study examined whether any sexual differences exist in the feathers of jungle crows, using electron microscopy and high performance liquid chromatography (HPLC).

Adult jungle crows of both sexes were captured from January 2005 to November 2006 at Niiza city, with permission of the Saitama prefecture (Permit to capture of wild animals No. 01-02), and kept in a laboratory animal facility. The sex of each bird was confirmed by gonad examination after dissecting. The secondary feathers (the feathers that adhere to the ulna) were removed from each individual under anesthesia with pentobarbital $(35 \mathrm{mg} / \mathrm{kg})$ and the feathers were washed under running water and stored under dry cool conditions until analysis.

First, we used electron microscopy to determine the

\footnotetext{
* Correspondence to: Sugita, S., Laboratory of Function and Morphology, Department of Animal Science, Faculty of Agriculture, Utsunomiya University, 350, Mine-machi, Utsunomiya City, Tochigi 321-8505, Japan.

e-mail: sugita@cc.utsunomiya-u.ac.jp
}

microstructure of the feather barbules. For this study, five adult males and four adult females were used. We cut the upper $0.5 \mathrm{~cm}$ of the barb of 5 barbs from the secondary feathers of each bird. The feather barbs were immersed in $0.1 \%$ Tween-20 for $1 \mathrm{hr}$ followed by $0.25 \mathrm{M}$ sodium hydroxide. These barbs were fixed with $1 \%$ osmic acid solution for $24 \mathrm{hr}$ after immersion in $2.5 \%$ glutaraldehyde for $48 \mathrm{hr}$. They were dehydrated in ethanol $(70 \%, 80 \%$ and $90 \%)$ and then rinsed in 90\% ethanol: $90 \%$ acetone $(1: 1 \mathrm{v} /$ v), followed by 90,95 and $100 \%$ acetone. They were then immersed in Epon 812 with absolute acetone (1:1 v/v, overnight), followed by Epon 812 (overnight). The immersion in Epon 812 was repeated twice under vacuum in a desiccator for 3-4 hr. The barbs were placed into moulds and the blocks were cured in an oven at $60^{\circ} \mathrm{C}$ for $24 \mathrm{hr}$. Ultrathin sections $70 \mathrm{~nm}$ thick were cut with an Ultracut S ultramicrotome (Reichert-Jung Optische Werke AG, Wien, Austria) equipped with a diamond knife (Diatome, Switzerland). Sections were collected with 200 mesh copper grids, stained with $2 \%$ uranyl acetate in $50 \%$ ethanol, and poststained with $0.2 \%$ lead citrate. Stained sections were observed using a transmission electron microscope (H-800, HITACHI, Japan) under an accelerating voltage of $100 \mathrm{kV}$ at $\times 6,000$ 7,000 magnification. We measured the density of melanin granules only in the distal barbules. To calibrate the images, we scanned these micrographs at 300 d.p.i. using an Epson GT-X700 flatbed scanner and Canvas software (ACD Systems international, Saanichton, BC, Canada).

Second, we used high performance liquid chromatography (HPLC) to determine melanin concentrations in the iridescent feather color of crows. To determine the eumelanin concentration, 15-30 mg of iridescent feather barbules in 11 adult males and 10 adult females were trimmed, and homogenized in water $(10 \mathrm{mg} / \mathrm{m} l)$. One hundred $\mu l$ feather homogenate was added to $850 \mu l 1 \mathrm{M} \mathrm{K}_{2} \mathrm{CO}_{3}$, and oxidized with $50 \mu l 30 \% \mathrm{H}_{2} \mathrm{O}_{2}$. The resulting oxidation product (pyrrole2, 3, 5-tricarboxylic acid, PTCA) derived from eumelanin 

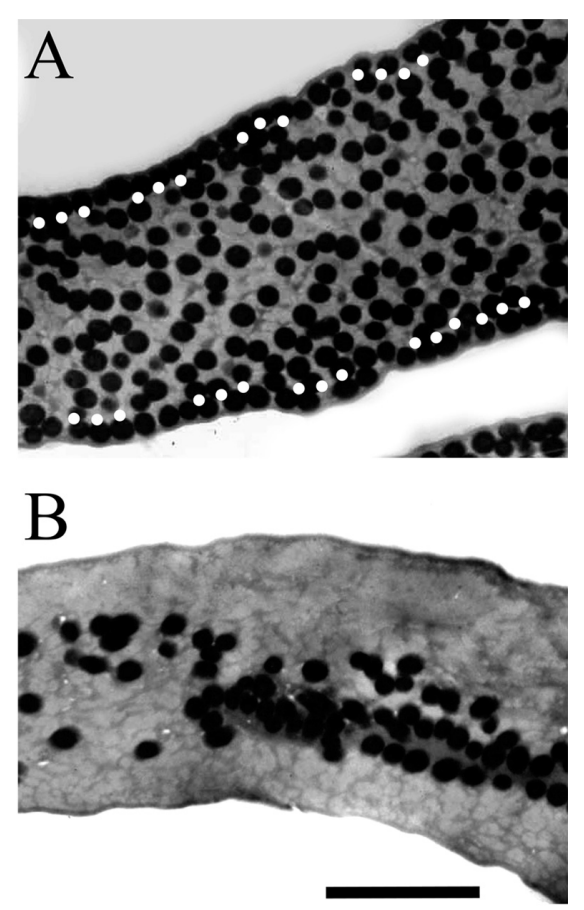

Fig. 1. Transmission electron micrographs of a barbule cross-section. (A) Male, (B) Female. A single-layer arrangement of melanin granules under the keratin is seen in the male. The iridescent color in crow's feather is produced by a single-layer arrangement. Broken dot lines show boundary between a single layer melanin granules area and irregular melanin granules area. Scale bar indicate $2 \mu \mathrm{m}$.

was analyzed with HPLC [12] with ultraviolet detection. Pheomelanins were assessed by hydrolyzing $100 \mu l$ feather homogenate with $500 \mu l 57 \%$ hydriodic acid at $130^{\circ} \mathrm{C}$ in the presence of $\mathrm{H}_{3} \mathrm{PO}_{2}$ for $24 \mathrm{hr}$, and subsequently analyzing the product (4-amino-3-hydroxyphenyalanine; 4-AHP) using HPLC with electrochemical detection [11].

We used the Mann-Whitney $U$ tests to analyze barbules melanin granule density (male: $n=48$, female: $n=48$ ) and feathers melanin concentration (male: $n=11$, female: $n=10$ ).

The spherical melanin granules were packed beneath a keratin cortex in the cross-section of each barbule. In males, the melanin granules were in a single-layer array just below the keratin cortex (Fig. 1A). The density of melanin granules (Fig. 2A) in the cross-section of the distal barbules was significantly higher in males $\left(8.2 \pm 2.5\right.$ granules/ $\left.\mu \mathrm{m}^{2}\right)$ than in females $\left(4.9 \pm 2.3\right.$ granules $\left./ \mu \mathrm{m}^{2}\right)$. The melanin granule density between males and females was significantly different (Mann-Whitney $U$-test, $z=5.97, P<0.01$ ). High concentrations of eumelanin were found: males' feathers contained an average of $1373 \pm 179 \mathrm{ng}$ eumelanin/mg feather, whereas females' feathers contained significantly lower concentrations of $1096 \pm 194 \mathrm{ng}$ eumelanin/mg feather (Mann-Whitney $U$-test, $z=2.90, P<0.01$ ) (Fig. 2B, Fig. 3). The concentration of pheomelanin was below the detection lim-
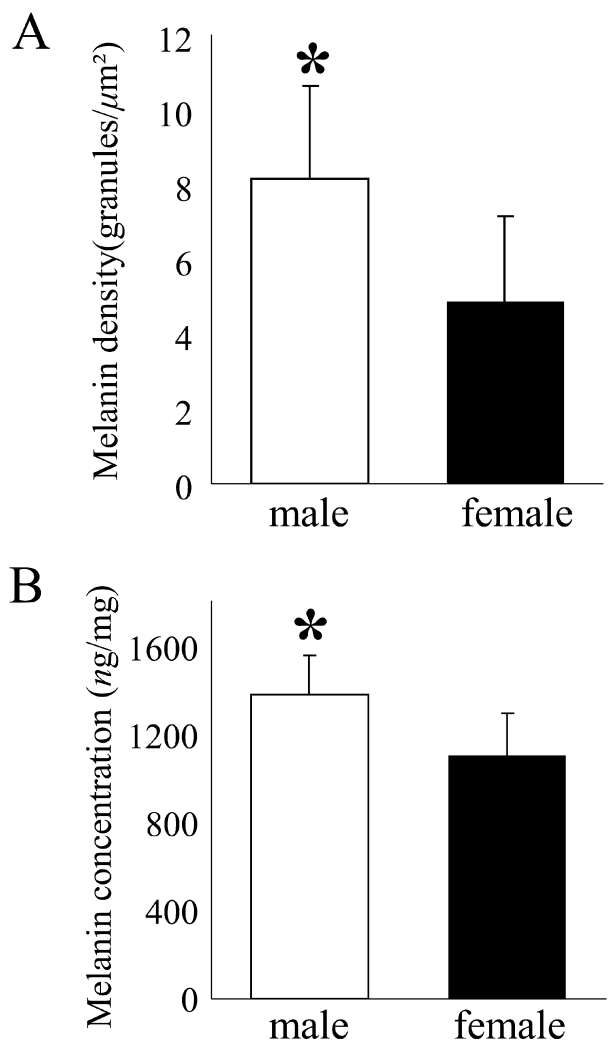

Fig. 2. Mean ( \pm SE) density of melanin granules (A) and concentration of eumelanin (B) found in secondary feathers from male and female Jungle crows. $* P<0.01$

its in this study.

In male crows, the iridescent color is produced by the single layer and high density of melanin. A single layer of melanin granules that produces oily red, green, blue, and purple iridescent colors are likely to evolve relatively easily in birds with black plumage $[9,10]$. The iridescent feather color in icteridae is essentially due to light scattering, density of melanin, and single layer [10]. Jungle crows also show that their iridescent feather color is produced by melanin single layer and density. Particularly, the melanin single layer causes weak diffraction grating effecting by light, whereas because female have not single layer, it can't expect diffraction grating effecting (unpublished data). The diffraction grating has important role which is causes iridescence in jungle crows' feather. This mechanism at least partially explains why iridescence is seen in black plumage.

We used an improved method with alkaline $\mathrm{H}_{2} \mathrm{O}_{2}$ oxidation for advantage of characterizing synthetic and natural eumelanins. The alkaline $\mathrm{H}_{2} \mathrm{O}_{2}$ oxidation method is easier to perform than a $\mathrm{KMnO}_{4}$ oxidation method, and it is more sensitive for eumelanin detection [12]. This chemical analysis coupled with HPLC yielded the concentration of eumelanin in the crows' feathers, but no pheomelanin was detected. Thus, feather color in jungle crows is determined 


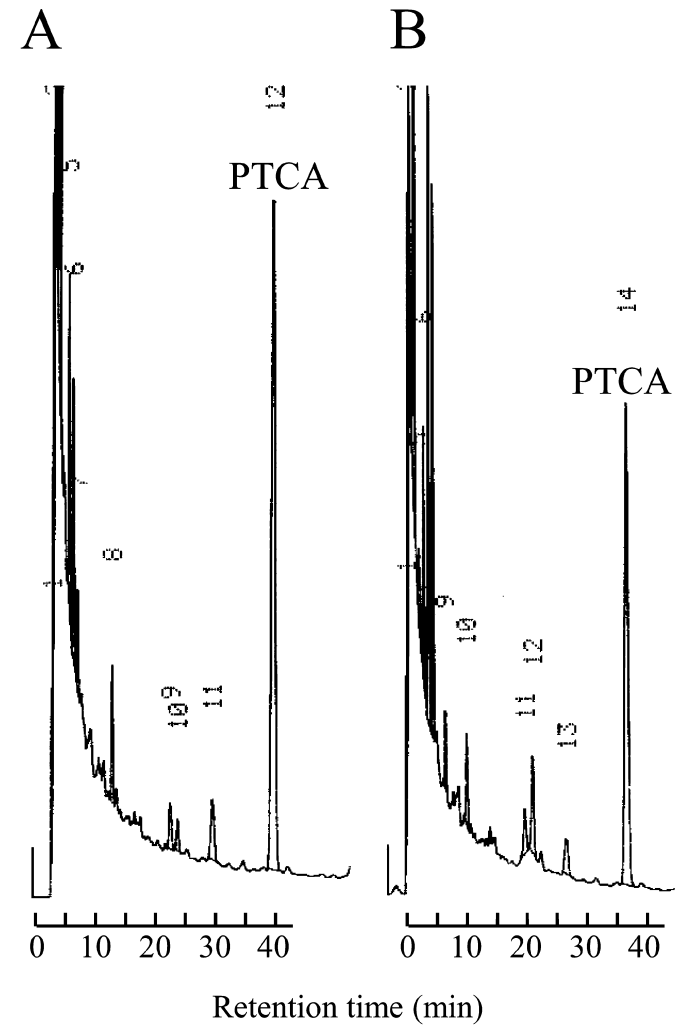

Fig. 3. HPLC analysis of hydrogen peroxide products in melanin-based iridescence in secondary feather. Male (A), Female (B). HPLC (JASCO AS-950, Japan) was performed in a CAPCELL PAK $\mathrm{C}_{18}$ column $(4.6 \times 250$ $\mathrm{mm})$ with a $0.01 \mathrm{M}$ potassium phosphate solution $(\mathrm{pH}$ 7.4 ) at the eluent at a flow rate $1.0 \mathrm{ml} / \mathrm{min}$. PTCA: pyrrole-2,3,5-tricarboxylic acid.

by eumelanin concentration and its concentration was higher in male than that in female. We used this improved method that extract only melanin from homogenized tissues. To understand the roles of feather color of both sexes, we need to compare melanin pigmented between the crows and other animals, regardless of the species and the shape of melanin. In eumelanin concentration, mammal was reported sexual differences in their black hair. Dorsal black hairs (wild type) of female house mice contain 1,600 $\mathrm{ng}$ eumelanin/mg of hair, and those of males contain 1,500 $\mathrm{ng}$ eumelanin/mg hair [3]. However, melanin concentration of house mice was analyzed by method of $\mathrm{KMnO}_{4}$ oxidation. As the used method is different, we cannot compare directly. The relation between $\mathrm{KMnO}_{4}$ oxidation and alkaline $\mathrm{H}_{2} \mathrm{O}_{2}$ oxidation is a good correlation and PTCA values obtained by alkaline $\mathrm{H}_{2} \mathrm{O}_{2}$ oxidation showed approximate twofold increases in the yield compared with $\mathrm{KMnO}_{4}$ oxidation [12]. This shows that the conversion factor of eumelanin in $\mathrm{H}_{2} \mathrm{O}_{2}$ oxidation is $1 / 2$ of $\mathrm{KMnO}_{4}$ oxidation. Thus the result of eumelanin concentration in crow feather became half number. Then, compared with mice, crows have not differences with mice in concentration of eumelanin; hence crows' feather color is similar to the black hair of mice. In the breast feathers in eastern bluebirds [5] and chestnut throat feathers in European barn swallows [7], these species' the males have higher concentration of total melanin than that those of the females. However, unlike crows, these species also have pheomelanin in their feathers, which combines with eumelanin and influences feather coloring. In the case of crow feather color is influenced by only eumelanin concentrations. The strong light absorption of the melanin pigments will further enhance the intensity of feather color [4]. The higher eumelanin concentration in the feathers of males may enhance the intensity of iridescent coloring by absorbing the unwanted complementary color. Thus, the iridescent color is more perceptible in a male's feather than that of a female.

We thank Prof. K. Sugahara for offering laboratory equipment and Assistant Prof. M. Aoyama for valuable advice. This work was supported by the Japan Society for the Promotion of Science (JSPS) grant (No. 16380185 (B)).

\section{REFERENCES}

1. Fox, H. M. and Vevers, G. 1960. The Nature of Animal Colors. Macmillan. New York.

2. Hasse, E., Ito, S. and Wakamatsu, K. 1992. Melanin concentrations in feathers from wild and domestic pigeons. J. Heredity. 83: 64-67.

3. Hirobe, T., Wakamatsu, K. and Ito, S. 2007. The eumelanin and pheomelanin contents in dorsal hairs of female recessive yellow mice are greater than in male. J. Dermatological Sci. 45: 55-62.

4. Kinoshita, S. and Yoshioka, S. 2005. Structural colors in nature: The role of regularity and irregularity in the structure. Chem. Phys. Chem. 6: 1442-1459.

5. McGraw, K.J., Wakamatsu, K., Ito, S., Nolan, P. M., Jouventina, P., Dobson, F.S., Austic, R. E., Safran, R. J., Siefferman, L. M., Hill, G. E. and Parker, R. S. 2004a. You can't judge a pigment by its color: carotenoid and melanin content of yellow and brown feathers in swallows, bluebirds, penguins, and domestic chickens. Condor 106: 390-395.

6. McGraw, K.J. and Wakamatsu, K. 2004b. Melanin basis of ornamental feather colors in male zebra finches. Condor 106: 686690.

7. McGraw, K. J., Safran, R. J., Evans, M. R. and Wakamatsu, K. 2004c. European barn swallows use melanin pigments to color their feathers brown. Behav. Ecol. 15: 889-891.

8. Prota, G. 1992. Melanins and Melanogenesis. Academic Press. San Diego. CA.

9. Prum, R.O. 2006. Anatomy, physical, and evolution of structural colors. pp. 295-353. In: Bird Coloration, vol. 1 Mechanisms and Measurements (Hill, G.E. and McGraw, K.J. eds). Harvard University Press, Boston.

10. Shawkey, M.D., Hauber, M. E., Estep, L. K. and Hill, G. E. 2006. Evolutionary transitions and mechanisms of matte and iridescent plumage coloration in grackles and allies (Icteridae). J.R.Soc.Interface. 3: 777-786.

11. Wakamatsu, K., Ito, S. and Rees, J.L. 2002. The usefulness of 4amino-3 hydroxyphenylalanine as a specific marker of phaeomelanin. Pigment Cell Res. 15: 225-232.

12. Wakamatsu, K., Ito, S., Niki, Y., Hirobe, T. and Brilliant, M. 2008. Reexamination of microanalytical methods for melanin assay. Pigment Cell Melanoma Res. 21,251 (Abstr.). 